\title{
Diseño de un sistema alternativo para el tratamien- to de aguas residuales urbanas por medio de la técnica de lombrifiltros utilizando la especie Eise- nia foetida
}

\author{
Jacipt Alexander Ramón, * José Alejandro León y Nelson Castillo
}

Programa de Ingeniería Ambiental, Facultad de Ingenierías y Arquitectura, Universidad de Pamplona. Ciudad Universitaria, Pamplona.

*Autor para correspondencia: jacipt@unipamplona.edu.co

\section{Resumen}

La presente investigación tuvo como objetivo diseñar y construir un sistema sobre tratamiento de aguas residuales para la ciudad de Pamplona utilizando como base teórica el sistema de tratamiento Tohá creado por el Dr. Jose Tohá de la Universidad de Chile, proponiendo una serie de procesos que permiten el saneamiento de las aguas servidas después de su uso con fines domésticos, industriales o comerciales, para poder ser vertidas finalmente a cuerpos de agua naturales. Este proceso incluye un tratamiento con lombrifiltros (aserrín y Eisenia foetida), por donde las aguas residuales pasan seguidamente de una cama de carbón activado filtrando y una cama de grava de piedra, permitiendo oxigenar el agua a partir del goteo que existe entre las camas. Finalmente, el efluente sigue a una cama de piedra de rio de gran tamaño y a un decantador para realizar la toma de muestras. De esta manera se obtiene agua tratada con baja carga orgánica dentro de los parámetros que establece la Comisión de Regulación de Agua Potable y Saneamiento Básico CRA en Colombia.

Palabras clave: lombrifiltro, Eisenia foetida, tratamiento de aguas, cinética.

Editora: Pataquiva-Mateus, A. Y.

Citation: Ramón, J., León, J. y Castillo, N. (2015). Evaluación de un humedal artificial de flujo subsuperficial para el tratamiento de aguas residuales domésticas. Revista Mutis 5(1); pag 46-54.

Received: May 10, 2015; Accepted: May 25, 2015; Published on line: June 30, 2015

\footnotetext{
Copyright: $\odot 2014$ Ramón et al. This is an open-access article, which permits unrestricted use, distributions and reproduction in any medium, provided the original author and source are credited.
}

Competing Interests: The authors have no conflict of interest.
Design of an alternative system for the treatment of urban waste water by means of the technique of earthworm-filters using the Eisenia foetida specie

\begin{abstract}
The present research aimed to design and build a system on treatment of wastewater for the city of Pamplona used as a theoretical basis the Tohá treatment system by Dr. José Tohá from the University of Chile, proposing a series of processes that allow the improvement of the wastewater after use
\end{abstract}


for domestic, industrial or commercial purposes. Wastewater passed through a treatment with earthworm-filters (saw dust and Eisenia foetida), followed by a bed of activated carbon and a bed of gravel stone, allowing oxygenation and filtration water due to dripping between beds. Subsequently, wastewater pass through a bed of river stone of large size; and finally, water decants into a collector for sampling. In this way, water treated with low load organic, within the parameters established by the Committee on regulation of drinking water and basic sanitation in Colombia (CRA), was obtained.

Keywords: earthworm-filters, Eisenia foetida, water treatment, kinetic.

\section{Introducción}

La creciente demanda de los recursos hídricos ha disminuido en las últimas décadas su disponibilidad y deteriorado su calidad, lo que causa un gran impacto en la disminución del acceso a agua segura en la mayoría de las áreas rurales y periféricas urbanas de los países en desarrollo.

Cabe resaltar que cualquier cuerpo de agua es capaz de asimilar cierta cantidad de contaminantes sin ser afectado seriamente debido a los factores de dilución y autopurificación. Hoy en día la presión que reciben las fuentes de agua es creciente, debido al aumento de actividades como agricultura, ganadería, industria, comercio, utilización de agua para regadíos de parques, áreas urbanas, baños sanitarios, etc. Estas actividades producen contaminación por aguas servidas, lo que trae como consecuencia el incremento de enfermedades, principalmente gastrointestinales; y la ausencia del preciado recurso para las actividades de subsistencia como la agricultura y la ganadería.

El sistema Tohá es una alternativa amigable con el medio ambiente (Laws, 2003), que consiste en una serie de procesos que tienen como objetivo eliminar los contaminantes físicos, químicos y biológicos que poseen tanto las aguas servidas como los residuos industriales líquidos. Con el tratamiento se obtiene agua limpia (o efluente tratado) y un residuo sólido denominado lodo o biosólido, que se debe disponer de una manera segura para evitar contaminación y malos olores, contribuyendo a la recuperación del recurso y a disminuir los impactos en la salud y el ambiente (Kusanovic, 2007).

\section{Marco Teórico}

\section{Agua contaminada}

Se le denomina contaminación natural a la originada por restos animales y vegetales y por minerales y sustancias que se disuelven cuando los cuerpos de agua atraviesan diferentes terrenos. Por otro lado la contaminación artificial es aquella que va apareciendo a medida que el hombre comienza a interactuar con el medio ambiente y surge con la inadecuada aglomeración de las poblaciones y como consecuencia del exponencial aumento del sector industrial (Departamento de Sanidad del Estado de Nueva York, 2002).

Actividades tales como lavado de automóviles, higiene, limpieza, refrigeración y procesos industriales en general, hacen uso de grandes cantidades de agua con diferentes niveles de contaminación, por lo que es necesario desarrollar nuevos mecanismos para su tratamiento (Departamento de Sanidad del Estado de Nueva York, 2002).

\section{Aguas residuales}

Son llamadas aguas residuales las que resultan después de haber sido utilizadas en domicilios, fábricas o actividades ganaderas, entre otras actividades. Las aguas residuales conllevan grasas, detergentes, materia orgánica, residuos de la industria, además de herbicidas, plaguicida y sustancias tóxicas.

Estas aguas residuales, antes de volver a la naturaleza, deben ser depuradas. Para ello se conducen a las plantas o estaciones depuradoras, donde se realiza el tratamiento más adecuado para devolver el agua a la naturaleza en las mejores condiciones posibles. Todavía existen muchos pueblos y ciudades de nuestro país que vierten sus aguas residuales directamente a los ríos, sin depurarlas. Esta conducta ha provocado que la mayoría de los seres vivos que vivían en esos ríos hayan desaparecido.

\section{Beneficios ambientales del tratamiento de aguas re- siduales domésticas}

Según Freeman (1982), los beneficios ambientales se pueden clasificar en: aquellos que son percibidos por las personas y aquellos que no lo son. Cada categoría mencionada por Freeman puede describir cómo se perciben estos beneficios dentro de un sistema de 
mercado, por ejemplo la gran disponibilidad y bajos precios de bienes para el consumidor; o a través del incremento de la disponibilidad de los bienes y servicios que no son normalmente comprados en el mercado; por ejemplo: mejora en las condiciones del ambiente como la visibilidad, alta calidad del agua para la recreación, etc. La primera categoría es llamada "el efecto mercado" y la segunda el "efecto de no mercado".

Para Freeman (1982), existen tres etapas en la producción de beneficios para el mejoramiento del ambiente, los cuales se producen a través de políticas de control de la contaminación. La primera etapa es casi completamente no económica en naturaleza, porque involucra una variedad de procesos físicos, químicos y biológicos; la segunda etapa involucra las interfaces entre la etapa no económica y la producción de los beneficios; mientras que la tercera etapa es totalmente económica porque involucra teoría de demanda y producción y la teoría de valor económico (Beascohechea, 2005).

\section{Materiales Y Métodos}

\section{Descripción del lombrifiltro}

Es un sistema de tratamiento de aguas servidas basado en la tecnología de lombrices (Eisenia foetida), que por sus características físicas o estructurales tiene alta eficiencia en la remoción de materia orgánica y organismos patógenos. Este es un biofiltro compuesto de cuatro estratos o lechos filtrantes de diversos materiales, a través del cual se hace pasar el agua servida. La capa superior consiste en un material orgánico con un gran número de microorganismos y lombrices. Cuando el agua residual pasa a través del lecho filtrante o sustrato, estos organismos absorben y digieren la materia orgánica eliminando su principal contaminante que son los residuos sólidos patógenos presentes en las aguas servidas (Fernandez, 2011).

El lombrifiltro fue construido como sigue: se tiene un tanque alimentador de $48 \mathrm{~m}^{3}$, el cual vierte al biofiltro con un volumen de agua de $100 \mathrm{ml} / \mathrm{min}$ en un tiempo de media hora, pasando el agua por una tubería de PVC de 12" de diámetro de acueducto, por tres llaves de válvulas de corte conectadas en serie una de la otra, por lo cual una vez el agua pasa por las llaves de corte, esta se dirige al biofiltro, repartiéndose en tres tubos de PVC agujerados para permitir un caudal continuo y estable, de esa manera podrá ser repartida homogéneamente el agua servida al sustrato del lombrifiltro.

Existe un lecho filtrante de aserrín con lombriz, seguido de un estrato de antracita que permite eliminar las impurezas presentes en el agua, para posteriormente ser filtrada por un lecho de triturado de piedra de río, para que finalmente esta agua servida se siga filtrando por un último lecho de piedra de río. Estas piedras ayudarán a obtener un líquido más limpio y tratado con menos impurezas y más claro para poder ser entregado a las cuencas de los ríos, dentro de los parámetros de la normatividad colombiana (Comisión de Regulación de Agua Potable y Saneamiento Básico - CRA)

Por consiguiente, una vez el agua es filtrada por cada uno de los lechos del lombrifiltro, puede ser utilizada para el riego de cultivos, siendo esta apta para el consumo humano. De otra manera, estos lechos sin lugar a dudas proveen soporte, aireación y un excelente manejo ambiental al sistema, en el cual el agua pasa a través del biofiltro por gravedad y emerge claramente y sin materia orgánica.

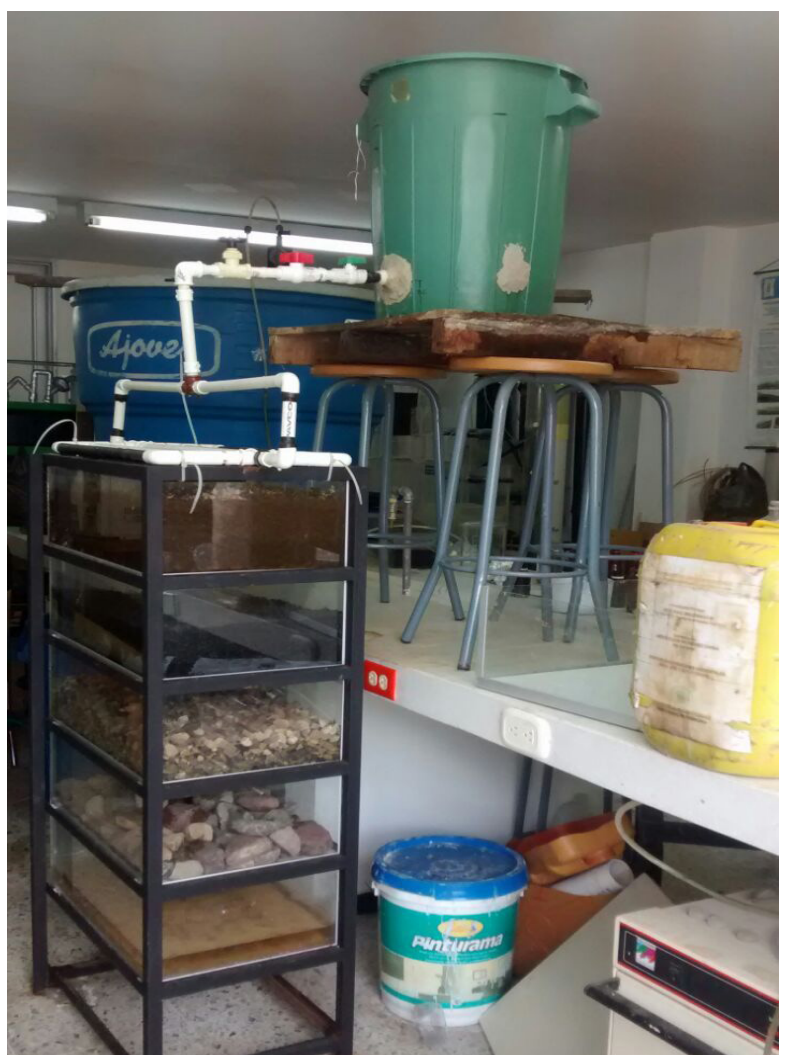

Figura 1. Montaje del lombrifiltro 


\section{Toma de muestras y descripción analítica}

La metodología utilizada para el proceso de validación de los métodos analíticos DQO, DBO5, sólidos suspendidos totales (SST) y sólidos suspendidos volátiles (SSV) está basada en el "procedimiento general de validación de metodologías analíticas" propuesto por la guía de laboratorio de química ambiental y bioprocesos de la Universidad de Pamplona.

El punto de muestreo que se escogió para la toma de muestras es el colector que vierte las aguas residuales urbanas, que se encuentra ubicado en las coordenadas del punto $X=1307577 ; Y=1158341$, frente a la Estación de Policía y al Terminal de Transporte del municipio de Pamplona, Norte de Santander (Colombia).

Se propuso un muestreo simple con el fin de identificar la fuente y evaluar los efectos potenciales en los procesos de tratamiento, determinando las característica de descarga instantánea a diferentes horas del día (2:00, 4:00 y 6:00 p. m.) en el punto de muestreo. A las muestras obtenidas de este punto, se les realizó una caracterización de los parámetros fisicoquími$\cos \left(\mathrm{DQO}, \mathrm{DBO}_{5}, \mathrm{SST}, \mathrm{SSV}\right.$, conductividad eléctrica, $\mathrm{pH}$, temperatura, oxígeno disuelto), y se estableció como hora de recolección para el proyecto las 2:00 p. m., ya que esta hora arrojó en los análisis de laboratorio de control de calidad mayor carga orgánica y se comportó más estable en comparación con las otras horas.

\section{Diseño del lombrifiltro}

En el diseño del sistema piloto del lombrifiltro se tuvieron en cuenta los siguientes parámetros de diseño: caudal, velocidad, tiempo de retención hidráulico, profundidad, ancho y largo de la caja, y altura del sistema; parámetros que intervinieron en el proceso para reducir los índices de contaminación de las aguas residuales urbanas (Orozco, 2014).

Los lechos fueron clasificados de la siguiente forma: aserrín con lombriz (dos lechos), seguidos de un lecho con antracita, pasando luego por un lecho de triturado de piedra, para finalmente colocar un lecho de piedra de río como se observa en la figura 2; permitiendo filtrar y oxigenar este líquido sin llegar a generar malos olores. Debido a que el proceso es aerobio, el agua filtrada por cada uno de los lechos filtrantes se recolecta en el último recipiente, que es usado para la toma de muestras de laboratorio.

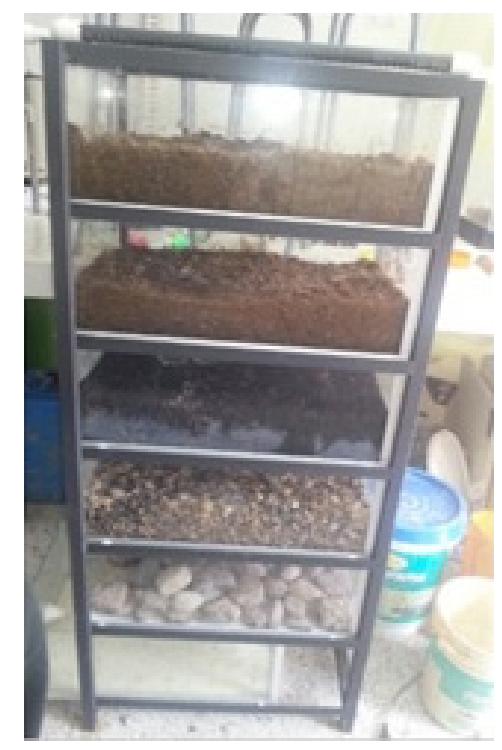

Figura 2. Lombrifiltro con cada lecho. De arriba a abajo: aserrín + lombriz (dos primeros cajones), antracita, triturado y bolones de piedra

\section{Diseño del lombrifiltro en bandejas de vidrio}

Cada bandeja contiene una capa de $10 \mathrm{~cm}$ de alto de cada material (aserrín, antracita y grava), excepto las piedras de río que contienen una altura de $7 \mathrm{~cm}$ debido a su gran tamaño y peso, que pueden ocasionar daño a la estructura del lombrifiltro. Inicialmente se alimentó el sistema del lombrifiltro con un caudal mínimo de 0,05 I/min el cual se determinó para poder suministrar al lombrifiltro un caudal continuo durante 12 horas con el fin de obtener una óptima eficiencia en el sistema. Con el propósito de evaluar el grado de remoción de materia orgánica, se caracterizaron los efluentes a través de los siguientes parámetros: demanda química de oxigeno (DQO), sólidos suspendidos totales y volátiles (SST - SSV), oxígeno disuelto, conductividad, $\mathrm{pH}$ y temperatura; de igual manera se realizaron análisis de $\mathrm{pH}$ al sustrato de la lombriz manteniéndose este entre 7 y 7,5 sin que llegase a alterar las muestras del agua o algún daño a la lombriz misma.

El sistema se alimentó diariamente con caudal continuo por un período de 90 días, el cual alcanzó el mayor rendimiento en el proceso de remoción de materia orgánica en un 92,06 \%; indicando que el proceso ya se encuentra en condiciones estables y de mayor remoción. El rendimiento del lombrifiltro se estipuló por el sustrato de entrada menos el de salida como $\mathrm{DQO}$ Y $\mathrm{DBO}_{5}$, y la producción de biomasa como SSV, después de estar este en estabilidad de los muestreos 
cada $0,25,0,5,1,2,3,6,12$ y 24 horas; cada diez horas se le agregaba agua sin tratar al recipiente de suministro, de las doces horas que tardaba en agotársele a este sistema por gravedad el agua a filtrar.

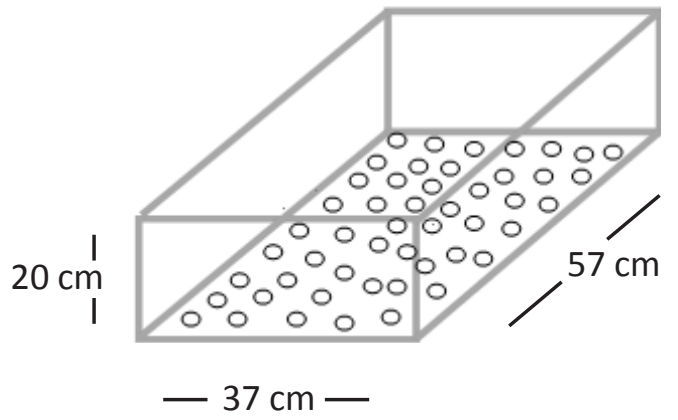

Figura 3. Dimensiones de cada uno de los cajones que conforman el lombrifiltro

La tabla 1 indica el valor del volumen de cada estrato dispuesto en el lombrifiltro, que cuenta con cajones de $42180 \mathrm{ml}$ cada uno.
Tabla 1. Volúmenes ocupados por los estratos que conforman el lombrifiltro construido

\begin{tabular}{|c|c|}
\hline ESTRATO & VOLUMEN (ml) \\
\hline Aserrín & 15817,50 \\
\hline Antracita & 14235,75 \\
\hline Grava & 11335,88 \\
\hline Piedra & 8857,80 \\
\hline
\end{tabular}

La selección de los caudales se realizó mediante las diferentes aperturas de la válvula de entrada de agua al lombrilfiltro, y se especifican en la tabla 2.

Tabla 2. Tiempo de retención y caudales utilizados en el modelado y simulación en Matlab ${ }^{\circledR}$

\begin{tabular}{|c|c|}
\hline NOMENCLATURA & CAUDAL (I/ $\mathbf{m i n})$ \\
\hline Q1 & 0,050 \\
\hline Q2 & 0,147 \\
\hline Q3 & 0,450 \\
\hline Q4 & 0,614 \\
\hline Q5 & 0,846 \\
\hline
\end{tabular}

Los tiempos de retención hidráulica (TRH) fueron medidos como el tiempo que demora el agua en pasar por el estrato e iniciar su aparición en el fondo de este, empleando cada uno de los caudales seleccionados.

Tabla 3. Tiempos de retención hidráulica para cada uno de caudales seleccionados.

\begin{tabular}{|c|c|c|c|c|c|}
\hline \multirow{2}{*}{ ESTRATO } & \multicolumn{5}{|c|}{ TIEMPOS DE RETENCIÓN } \\
& \multicolumn{5}{|c|}{ HIDRÁULICA (h) } \\
\cline { 2 - 6 } & TRH $_{\mathbf{1}}$ & TRH $_{\mathbf{2}}$ & TRH $_{\mathbf{3}}$ & TRH $_{\mathbf{4}}$ & TRH $_{5}$ \\
\hline Aserrín & 8,96 & 3,05 & 1,00 & 0,73 & 0,53 \\
\hline Antracita & 2,28 & 0,78 & 0,25 & 0,19 & 0,14 \\
\hline Grava & 3,25 & 1,10 & 0,36 & 0,26 & 0,20 \\
\hline Piedra & 2,81 & 0,96 & 0,31 & 0,23 & 0,17 \\
\hline
\end{tabular}

Los datos provenientes de las tablas 1,2 y 3 ; se utilizaron para modelar, simular y hallar el rendimiento del lombrifiltro por medio del programa Matlab ${ }^{\circledR}$.

\section{Constantes cinéticas y estequiométricas}

Los modelos utilizados para determinar las constantes cinéticas $k$ y $k s$ en el lombrifiltro, normalmente están compuestos por el submodelo hidráulico y balances de masa con términos de generación y remoción de masa afectados por constantes cinéticas, a través de los datos obtenidos en el laboratorio de control y calidad de la Universidad de Pamplona.

A partir, de la ecuación 1 , de remoción de sustrato de Lineweaver-Burk que describe la velocidad específica de utilización de sustrato. Se graficó la pendiente recta de regresión que estima $\mathrm{ks} / \mathrm{k}$ y la ordenada al origen $1 / k$. (McKinney, 1974). 


$$
\frac{1}{U}=\frac{i X}{S_{\circ}-S}=\frac{k_{S}}{k}=\frac{1}{S}+\frac{1}{k}
$$

$\mathrm{U}=\Theta$ tasa neta de remoción de sustrato (dias).

$\mathrm{X}=$ biomasa en el reactor (mg SSV/L).

$\mathrm{S}_{0}=$ Sustrato en el reactor ( $\mathrm{mg} \mathrm{DQO} / \mathrm{L}$ ).

$\mathrm{KyK}_{\mathrm{s}}=$ Constantes cinéticas. $\left(\mathrm{d}^{-1}\right)$.

En primer lugar, se obtienen los datos en una tabla de coeficiente cinético y estequiométrico, donde se determina el caudal 1 , tiempo de detención respecto al primer caudal (71,429 I/día) y el comportamiento de sustrato y biomasa en los cinco puntos de control.

De acuerdo a la figura 4 y a la tabla de coeficiente cinético y estequimétrico, se determinaron las constantes $k_{0}$ y $k_{m}$ de remoción de sustrato por medio de la ecuación de Lineweaver-Burk, obteniendo como dato de partida el coeficiente estequiométrico de remoción $Y=0.0002 x$

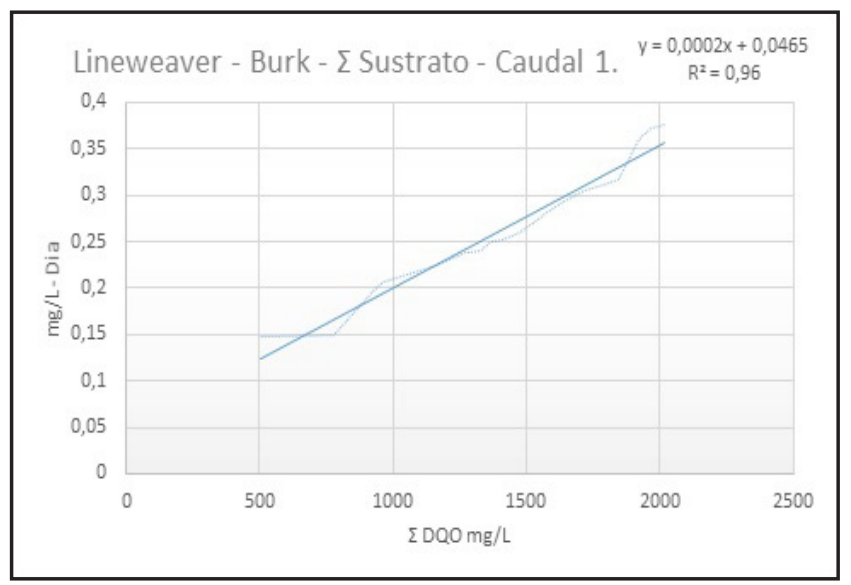

Figura 4. Recta de regresión para hallar K y Ks

Al mismo tiempo, de acuerdo a la figura 5 y la tabla número 4 , en el caudal 1 se determinó las constantes $Y$ y $1 / \theta$ de crecimiento de biomasa por medio de la ecuación de Lineweaver-Burk, obteniendo como dato de partida el coeficiente estequiométrico de crecimiento $\mathrm{Y}=1.9735 \mathrm{x}$

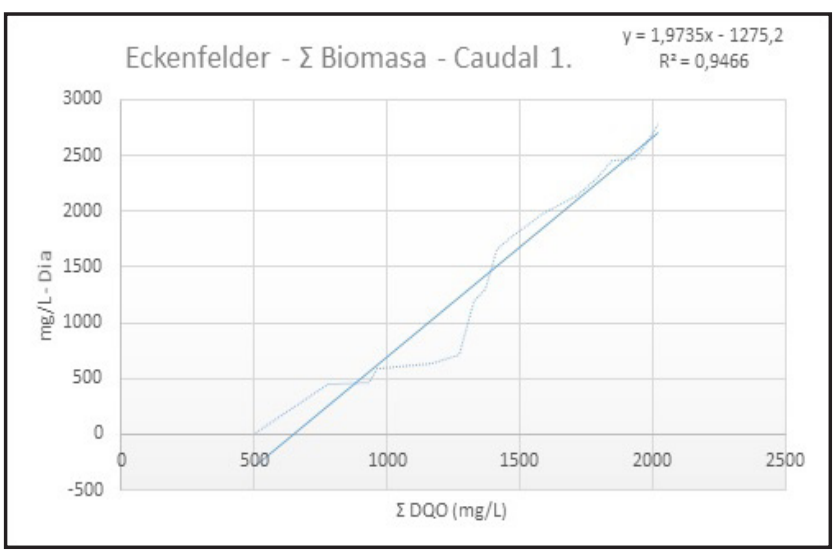

Figura 5. Recta de regresión para hallar Y y $1 / \theta$

Tabla. 5. Constantes de sustrato y biomasa

\begin{tabular}{|c|c|c|c|c|}
\hline \multirow{2}{*}{ CAUDAL } & \multicolumn{4}{|c|}{ CONSTANTES } \\
\cline { 2 - 5 } & $\mathrm{K}_{\mathrm{o}}=\mathrm{K}_{\mathrm{s}}$ & $\mathrm{K}_{\mathrm{m}}=\mathrm{k}_{\mathrm{e}}$ & $\mathrm{Y}$ & $1 / \theta$ \\
\cline { 2 - 5 } & $\left(\mathrm{d}^{-1}\right)$ & $\begin{array}{c}(\mathrm{mg} \\
\mathrm{DQO} / \mathrm{l}) \\
\left(\mathrm{d}^{-1}\right)\end{array}$ & $\begin{array}{c}(\mathrm{mg} \mathrm{SSV} \\
/ \mathrm{mg} \\
\mathrm{DQO})\end{array}$ & $\left(\mathrm{d}^{-1}\right)$ \\
\hline $\mathrm{Q} 1$ & 21,51 & 0,043 & 0,563 & 1,575 \\
\hline $\mathrm{Q} 2$ & 30,00 & 0,033 & 0,498 & 1,375 \\
\hline $\mathrm{Q} 3$ & 34,28 & 0,371 & 0,414 & 1,272 \\
\hline $\mathrm{Q} 4$ & 37,34 & 0,060 & 0,235 & 1,138 \\
\hline $\mathrm{Q} 5$ & 39,55 & 0,022 & 0,218 & 1,047 \\
\hline
\end{tabular}

\section{Resultados y Discusión}

Con la utilización del lombrifiltro, se obtuvieron impactos positivos en la calidad del agua que se vierte a los cuerpos de agua o al subsuelo, en conclusión al tratamiento que es muy eficiente en la remoción de los contaminantes y microorganismos patógenos, presentando reducciones superiores al 90 \%. En mención de los análisis de resultados obtenidos en los parámetros fisicoquímicos del laboratorio como son $\mathrm{pH}$, temperatura, oxígeno disuelto, conductividad, demanda química de oxígeno (DQO), demanda biológica de oxigeno (DBO5), sólidos suspendidos totales (SST) y los sólidos suspendidos volátiles (SSV), se determinó la eficiencia del proceso y el buen funcionamiento del lombrifiltro.

La evaluación del rendimiento del sistema de lombrifiltro se determinó a partir del comportamiento de la DQO durante el proceso y los SSV como la de biomasa del sistema. 
En la figura 6 se puede observar el rendimiento del sistema de lombrifiltro, indicando un rendimiento máximo del 92,06 \% del sistema, por la sumatoria de los tiempos de retención hidráulicos vs. sustrato de salida del lombrifiltro, a comparación de otras tecnologías existentes en la remoción de sustrato como por ejemplo lodos activados, UASB, lagunas de estabilización y oxidación, humedales artificiales de flujo superficial y reactores discontinuos (RAS, 2000).

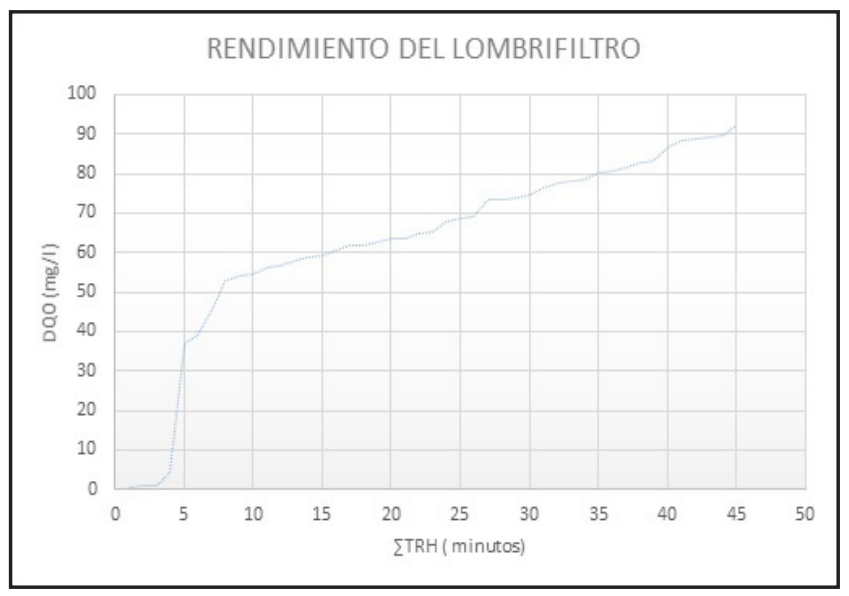

Figura 6. Rendimiento del lombrifiltro

Por otra parte, en la figura 7 se demuestra cómo disminuye la carga orgánica que ingresa al sistema del lombrifilro, obteniéndose como resultado la remoción de DQO en porcentajes con respecto a los tiempos de retención hidráulicos (TRH) trabajados anteriormente. Se puedo observar cómo el lombrifiltro consume casi en su totalidad la DQO de entrada dependiendo del TRH.

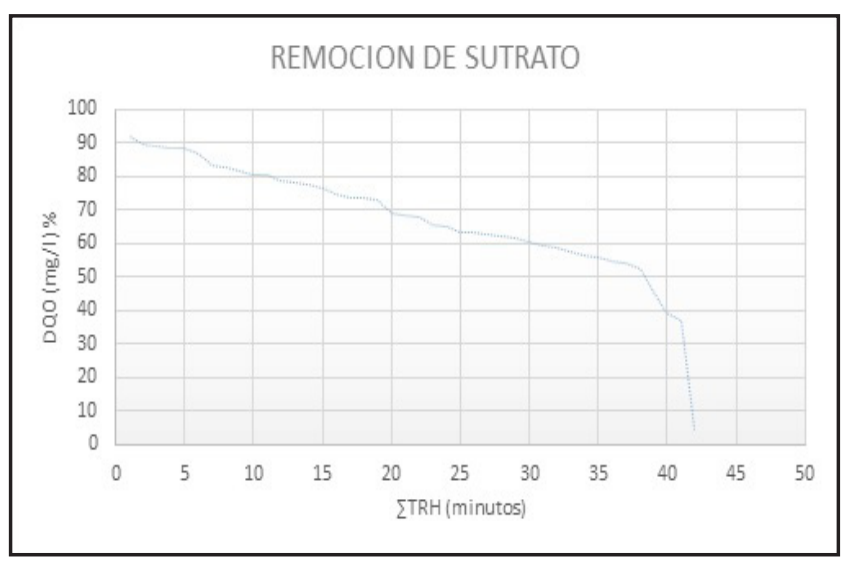

Figura 7. Remoción de sustrato

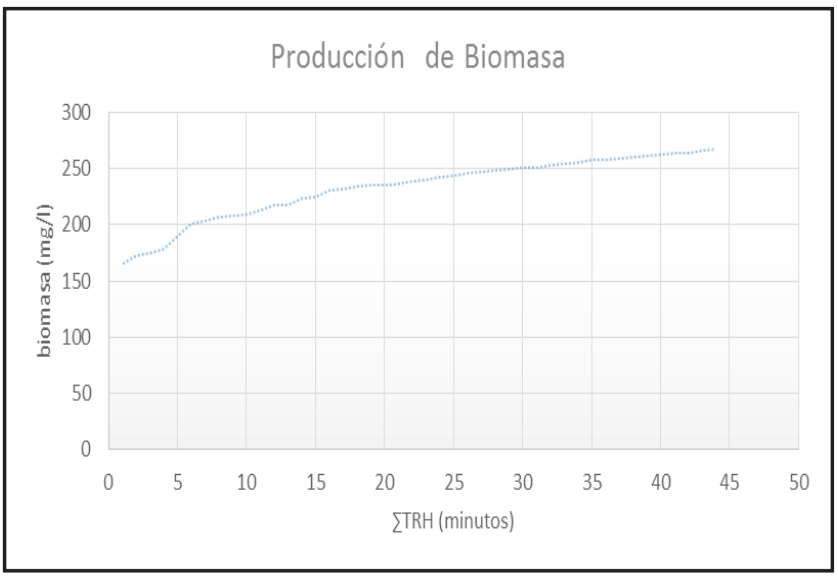

Figura 8. Producción de biomasa

Para evaluar la eficiencia del lombrifiltro, esta se determinó por medio de análisis de laboratorio de las muestras de agua residual urbana obtenidas a través del colector principal del municipio de Pamplona, midiéndose los parámetros fisicoquímicos (DQO, $\mathrm{DBO}_{5}$, SST, SSV), y la remoción de coliformes totales y fecales; y obteniéndose como resultado un sistema que cumple con más del $92 \%$ de eficiencia en la disminución de carga orgánica del efluente del lombrifiltro como se puede observar en la tabla 8.

Tabla 8. Porcentaje de DQO

\begin{tabular}{|c|c|c|}
\hline $\begin{array}{c}\text { DQO ENTRADA } \\
(\mathrm{mg} / \mathrm{L})\end{array}$ & $\begin{array}{c}\text { DQO SALIDA } \\
(\mathrm{mg} / \mathrm{L})\end{array}$ & $\begin{array}{c}\text { DQO } \\
\text { REMOVIDA (\%) }\end{array}$ \\
\hline 542 & 43 & 92,066 \\
\hline 771 & 81 & 89,494 \\
\hline
\end{tabular}

El tiempo de proceso para obtener el 92,066 \% fue de 182 días después del arranque del lombrifiltro, a una temperatura de entre 17 y $20{ }^{\circ} \mathrm{C}$, pH entre 6,6 y 8 , oxígeno disuelto $0,3-0,95 \mathrm{mg} / \mathrm{L}$, conductividad eléctrica entre $722-895 \mu \mathrm{s} / \mathrm{cm}$, DQO entre $542-771 \mathrm{mg} / \mathrm{L}$, SSV entre 162-245 mg/L, y SST entre 234-330 mg .

La eficiencia obtenida de $89,5 \%$ tardó un tiempo de 33 días empleando las condiciones antes mencionadas. Los métodos utilizados para el análisis fisicoquímico del agua residual urbana fueron realizados en el 
laboratorio de control de calidad y utilizando la guía de laboratorio química ambiental y bioprocesos. El lombrifiltro obtuvo una eficiencia del 92,1\% de remoción de materia orgánica a partir de la DQO.

Por consiguiente, la técnica de tratamiento de aguas residuales urbanas utilizando el lombrifiltro es una alternativa viable y estable. Cabe resaltar que el efluente obtenido en el lombrifiltro presenta unas propiedades libres de sólidos suspendidos dando cumplimiento a la normativa actual (Decreto 3930 de 2010 y Ley 373 de 1997) sin sólidos suspendidos apreciables (< $20 \mathrm{mg} / \mathrm{l}$ ), lo que facilita su manejo con fines de reuso.

\section{Obtención de las ecuaciones para lombrifiltro de biomasa y sustrato derivadas de la fórmula de Run- ge-Kutta para modelación y simulación en Matlab ${ }^{\circledR}$}

En la ecuaciones 2 y 3 derivadas de la fórmula de Runge-Kutta según autor (Romero, 2008), se observan los valores de las constantes cinéticas obtenidas de biomasa (SSV) y sustrato (DQO) para los caudales tratados, citando las constantes cinéticas de mayor remoción de sustrato en esta investigación, para el tratamiento de las aguas residuales urbanas por medio del lombrifiltro.

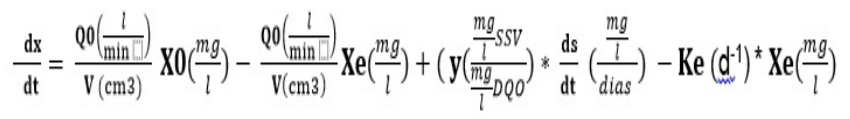

\section{Ecuación de sustrato}

$$
\frac{d s}{d t}=\frac{Q\left(\frac{l}{\min }\right)}{V(\mathrm{~cm} 3)} S 0\left(\frac{m g}{l}\right)-\frac{Q\left(\frac{l}{\min m}\right)}{V(\mathrm{~cm} 3)} S e\left(\frac{m g}{l}\right)-\frac{K S}{K e(\mathrm{mgDQ} 0 / \mathrm{L} * \mathrm{~d}-1)+S\left(\frac{m g}{l}\right) / X\left(\frac{m g}{l}\right)}
$$

\section{Conclusiones}

Se estudió el comportamiento del lombrifiltro por medio de un análisis de laboratorio a los parámetros DQO, $\mathrm{DBO}_{5}$ - SST - SSV, a la entrada y a la salida del sistema. De igual forma, se verificó que la carga del afluente era bastante alta en comparación con la del efluente, obteniendo una eficiencia del 92,06 \% de remoción de carga orgánica en el sistema de lombrifiltro.
El diseño del sistema piloto de tratamiento de aguas residuales con lombrifiltros tuvo como finalidad estudiar todos los factores que intervienen directamente en el sistema como son: temperatura, $\mathrm{pH}$, caudal, tiempo de retención hidráulico, constantes de remoción de sustrato y producción de biomasa, concentración de la carga orgánica, y la cantidad de lombrices ingresadas al estrato de aserrín, que permitieron altas eficiencias y rendimientos en el sistema.

De igual forma, la variedad de los lechos utilizados permitió obtener buenos resultados a nivel de laboratorio en la etapa de aserrín + lombriz, como también en la de antracita, que posibilitaron remover alta carga orgánica y sólidos en el agua residual urbana; determinando altas eficiencias al pasar por cada lecho del sistema, obteniéndose un agua más clara, sin olor y oxigenada. Cabe resaltar también que el triturado utilizado seguido de piedra de río, intervienen mucho en la remoción y oxigenación del agua filtrada.

El modelado y la simulación del sistema de lombrifiltros permitieron verificar, de acuerdo a las fórmulas de ecuaciones diferenciales de Runge-Kutta y halladas las constantes $\mathrm{K}$ de sustrato y biomasa a partir de las figuras, una tendencia decreciente (Orozco, 1974 y McKinney, 1974). Se determinó el comportamiento del sustrato al ser removido, mediante una cinética de orden variable en reactores de mezcla completa, consumiendo la DQO contraria a la de biomasa. De igual forma se utilizaron las ecuaciones de Orozco (1974) mostrando una figura creciente determinando que la biomasa genera SSV. A partir de estas constantes cinéticas se diseñó un software a través de la aplicación Guide de Matlab ${ }^{\circledR} 2012$ utilizando ecuaciones universales de balance materia.

\section{Referencias}

Banks, Ch. (1979). Algunos factores microbiológicos, bioquímicos, ecológicos y su aplicación al diseño y operación de procesos biológicos. ACISA, Cali.

Barnard, J. L., et al. (1972). "Design Optimization for Activated Sludge and Extended Aeration Plant", 6 th Int. Conf. of the IAWPR, Israel.

Beascoechea, Eduardo de Miguel; Curt F., María Dolores y Fernández González, Jesús. (2005). Reportaje sobre la depuración de aguas mediante filtros verdes en el medio rural. Madrid, España. 
Brey W. S. (1978). Physicalchemistry and its biological aplications, Academic Press.

Celis Hidalgo, José, Junod Montano, Julio y Sandoval Estrada, Marco. (2008). Recientes aplicaciones de la depuración de aguas residuales. Revista Theoria, Ciencia, Arte y Humanidades. 14(1), 17-25.

Departamento de Sanidad del Estado de Nueva York. (2002). Manual de tratamiento de aguas negras. México: Editorial Limusa, Noriega Editores.

Fernández, P, Raúl (2011), Sistema de aguas residuales a través de "Sistema Tohá". Recuperado de: www.sistematoha.cl

Freeman, Myrick. (1982). Air and water pollution control a benefit-cost assessment. New York: Wiley. $186 \mathrm{p}$.

Kusanovic, M. (2007). Proyecto construcción de planta de tratamiento de riles del Matadero Magallanes Ltda. Postulado por AVF Ingeniería Ltda. Aprobada por Conama con resolución exenta $N^{\circ} 150$ con fecha de diciembre del 2007. Punta Arenas, Chile.

Laws, J. E. A. (2003). Evaluación ambiental del sistema Tohá en la remoción de salmonella en aguas servidas domésticas. Magíster en Gestión y Planificación Ambiental. Universidad de Chile, Santiago. $92 p$.
Departamento de Sanidad del Estado de Nueva York. (2002). Manual de tratamiento de aguas negras. México: Editorial Limusa, Noriega Editores.

McKinney, R. E. (1974). Design and Operational Model for Complete Mixing Activated Studge System, Biotechnology and Bioengineering, Vol. 14.

Martínez, S. Rodríguez M. (2005). Tratamiento de aguas residuales con Matlab ${ }^{\circledR}$. Universidad Autonoma Metropolitana, México: Editorial Reverté S. A.

Orozco, A. (1977). Teoría de la remoción de sustrato soluble en plantas de lodos activados Bogotá: Revista Acodal.

Orozco, A. (2014). Bioingeniería de aguas residuales. Segunda edición, Bogotá: Editorial Acodal.

RAS. (2000). Reglamento técnico del sector de agua potable y saneamiento básico Título E. Minvivienda, Colombia.

Romero, Jairo Alberto. (2008). Tratamiento de aguas residuales teoría y principios de diseño. Bogotá: Editorial Escuela Colombiana de Ingeniería.

Universidad Austral de Chile. (2004). Estudio de factibilidad de la aplicación del sistema Tohá en la planta de tratamiento de aguas servidas de Valdivia. Facultad de Ciencias de la Ingeniería, Escuela de Ingeniería Civil en Obras Civiles. U. Austral de Chile, Valdivia. 